\title{
HYPERPLANE SECTIONS OF ARITHMETICALLY COHEN-MACAULAY CURVES
}

\author{
CHARLES H. WALTER
}

(Communicated by Wolmer V. Vasconcelos)

\begin{abstract}
We show that for every $r \geq 4$ there exists a $d_{r}$ such that for all $d \geq d_{r}$ a general set of $r$ points in $\mathbf{P}^{r-1}$ is not a hyperplane section of an arithmetically Cohen-Macaulay local complete intersection curve in $\mathbf{P}^{r}$. Explicit values for the bound $d_{r}$ are given. In particular, for $r \geq 12$ we have $d_{r}=r+3$, and this bound is exact.
\end{abstract}

One of the most important approaches to the study of projectively normal curves in $\mathbf{P r}^{r}$ is to study their hyperplane sections in $\mathbf{P}^{r-1}$. This applies both in the French school of curves in $\mathbf{P}^{3}$, and in Castelnuovo theory where the whole method is to study the numerical properties of curves by means of the numerical properties of their hyperplane sections. The reasons for this approach are that many of the algebraic and especially numerical properties of a projectively normal curve (including their Hilbert functions, minimal projective resolutions, degree, genus, etc.) are reflected in similar properties of the ideal of the hyperplane section; and since the hyperplane section is a set of points, it is in some ways easier to study.

In this context the following question was raised ([EEK]): is a general set of $d$ points in $\mathbf{P}^{r-1}$ always the hyperplane section of a projectively normal curve in $\mathbf{P}^{r}$ ? In [B] Ballico showed that for each $r \geq 7$ there exist a finite number of $d$ for which the answer is negative. But then he asked ([B] p. 145) whether for each $r \geq 7$ it is true that a general set of $d$ points in $\mathbf{P}^{r-1}$ is not the hyperplane section of a projectively normal curve for all $d \gg 0$.

In this paper we give an almost definitive answer to this last question of Ballico's. Our result is:

Theorem 1. For any integer $r \geq 4$, there exists an integer $d_{r}$ such that for all $d \geq d_{r}$ a general set of $d$ points in $\mathbf{P}_{k}^{r-1}$ (with $k$ a field of characteristic 0 ) is not the hyperplane section of any arithmetically Cohen-Macaulay local complete

Received by the editors September 15, 1993 and, in revised form, February 4, 1994.

1991 Mathematics Subject Classification. Primary 14H50.

Key words and phrases. Arithmetically Cohen-Macaulay, hyperplane section, local complete intersection, normal bundle.

Supported in part by NSA research grant MDA904-92-H-3009.

(C)1995 American Mathematical Society 
intersection curve in $\mathbf{P}_{k}^{r}$. Moreover, one may take $d_{4}=31, d_{5}=19, d_{6}=15$, $d_{7}=d_{8}=d_{9}=13, d_{10}=14, d_{11}=15$, and $d_{r}=r+3$ for $r \geq 12$.

We should note, as Ballico already pointed out in his paper, that Theorem 1 is false if $r=3$ ([CO] Corollary 16), or if the curves are not required to be arithmetically Cohen-Macaulay. For instance any set of $d$ points in $\mathbf{P}^{r-1}$ can be achieved as the hyperplane section of a nonsingular rational curve in $\mathbf{P}^{r}$ ([BM] Theorem 1.6). The local complete intersection property can also not be dropped (at least not completely) since the cone over any set of points in $\mathbf{P}^{r-1}$ is always an arithmetically Cohen-Macaulay curve. On the other hand, it is quite possible that the characteristic 0 condition might be unnecessary or that the particular values of $d_{r}$ given might not be the minimum possible if $3 \leq r \leq 11$. But for $r \geq 12$, the value $d_{r}=r+3$ is exact because in an accompanying paper ([W] Theorem 0.1 ) we show that a general set of $r+2$ points in $\mathbf{P}^{r-1}$ is the hyperplane section of a projectively normal curve of genus 2.

A particularly interesting corollary of the above theorem is:

Corollary 2. Let $r \geq 4$ and $d \geq d_{r}$ be integers, where $d_{r}$ is as in Theorem 1 . Then there exist at least two components of the Hilbert scheme of arithmetically Cohen-Macaulay curves of degree $d$ in $\mathbf{P}^{r}$ with the same genus and Hilbert function.

This corollary stands in marked contrast to the situation in $\mathbf{P}^{3}$, where the families of arithmetically Cohen-Macaulay curves of degree $d$ are in one-to-one correspondence with the different possible Hilbert functions of sets of $d$ points in $\mathbf{P}^{2}$ ([GP]). Thus although in $\mathbf{P}^{3}$ the Hilbert function of the hyperplane section is (almost) all that is necessary for the classification of arithmetically Cohen-Macaulay curves, this is not the case in $\mathbf{P}^{r}$ for $r \geq 4$.

The two components of the corollary are easy to describe. Both have the property that the hyperplane sections of the curves are zero-dimensional schemes of length $d$ with generic postulation. So if $s$ is the integer such that $\left(\begin{array}{c}s+r-1 \\ r-1\end{array}\right) \leq$ $d<\left(\begin{array}{c}s+r \\ r-1\end{array}\right)$, then the Hilbert function of the curves satisfies $H(n)=\left(\begin{array}{c}n+r \\ r\end{array}\right)$ if $n \leq s$, and $H(n)=d(n-s)+\left(\begin{array}{c}s+r \\ r\end{array}\right)$ if $n>s$. The genus is $g=s d+1-\left(\begin{array}{c}s+r \\ r\end{array}\right)$. One component contains cones over arbitrary sets of $d$ points in $\mathbf{P}^{r-1}$ with generic postulation. Theorem 1 implies that this component cannot contain any local complete intersection curves if $r \geq 4$ and $d \geq d_{r}$. The second component contains arithmetically Cohen-Macaulay local complete intersection curves with the same Hilbert function $H$. These are guaranteed to exist because of

Theorem 3 ([RR] Theorem 4.2(b)). Let $T \subset \mathbf{P}^{r-1}$ be a zero-dimensional scheme. Then there exists an arithmetically Cohen-Macaulay stick figure $C \subset \mathbf{P}^{r}$ whose proper hyperplane sections have the same Hilbert function as $T$.

Here a stick figure is a union of lines no three of which pass through a common point. Thus stick figures are local complete intersection curves.

The method used to prove Theorem 1 would apply to other classes of local complete intersection curves in $\mathbf{P}^{r}$ provided that the genera of curves in the class grow sufficiently fast as the degrees of the curves increase (see Theorem 5 below). 
Theorem 1 extends a result of Ballico ([B]) who showed that it held for certain values of $r, d$ but only for $r \geq 7$ and only for a finite number of values of $d$ for each $r$. On the other hand there was no characteristic 0 assumption in Ballico's proof.

We do not know under what conditions the stick figures of Theorem 3 can be smoothed. If $r=3$, the stick figure can be smoothed if and only if the Hilbert function of $T$ is of decreasing type. But for $r \geq 4$, it is still unclear whether the stick figure is smoothable even if $T$ is a general set of $d$ points in $\mathbf{P}^{r-1}$ except for certain special values of $d$. For instance, if $d=\left(\begin{array}{c}s+r-2 \\ r-1\end{array}\right)$, then the stick figures are degenerations of curves generated by the maximal minors of $s \times(s+r-2)$ matrices of linear forms.

The author would like to thank A. Geramita, J. Migliore, and B. Ulrich who brought Ballico's paper to his attention.

\section{The Proof of the Theorem}

We precede the proof of Theorem 1 with the proofs of several preliminary results.

Lemma 4. Let $C$ be a local complete intersection curve in $\mathbf{P}^{r}$ of degree $d$ and arithmetic genus $g$ over a field of characteristic 0 . Let $\mathscr{N}$ be the normal sheaf of $C$. If $H^{1}(\mathscr{N}(-1))=0$, then either $g \leq(2 d-4) /(r-3)$, or $C$ is a line.

Proof. By [H], Theorem III.7.11, $\mathscr{N}$ is a locally free $\mathscr{O}_{C}$-module of rank $r-1$, and $\Lambda^{r-1} \mathscr{N} \cong \omega_{C}(r+1)$. So $\mathscr{N}(-1)$ is locally free of rank $r-1$ of degree $\operatorname{deg}\left(\omega_{C}(2)\right)=2 d+2 g-2$. The Riemann-Roch theorem for locally free sheaves on curves then implies that

$$
\begin{aligned}
h^{0}(\mathcal{N}(-1))-h^{1}(\mathcal{N}(-1)) & =\operatorname{deg}(\mathcal{N}(-1))+(1-g) \operatorname{rk}(\mathcal{N}(-1)) \\
& =2 d-(r-3)(g-1) .
\end{aligned}
$$

We claim that if $H^{1}(\mathscr{N}(-1))=0$, then either $h^{0}(\mathscr{N}(-1)) \geq r+1$, or $C$ is a line. It is clear that the lemma follows from this claim.

To prove the claim, note that $H^{0}\left(\mathscr{T}_{\mathbf{P r}}(-1)\right)$ is of dimension $r+1$ with basis $\frac{\partial}{\partial X_{0}}, \ldots, \frac{\partial}{\partial X_{r}}$. There is a natural map $H^{0}\left(\mathscr{T}_{\mathbf{r} r}(-1)\right) \rightarrow H^{0}(\mathscr{N}(-1))$ whose kernel is $V=\left\{\xi \in H^{0}\left(\mathscr{T}_{\mathbf{P r}}(-1)\right) \mid \xi \cdot I(C) \subseteq I(C)\right\}$. If $V=0$, then $h^{0}(\mathscr{N}(-1)) \geq h^{0}\left(\mathscr{T}_{\mathbf{P} r}(-1)\right)=r+1$ as claimed. On the other hand if there is $0 \neq \xi \in V$, then we may assume $\xi=\frac{\partial}{\partial X_{0}}$. Then for $F \in I(C)$, if we write $F=\sum X_{0}^{i} F_{i}\left(X_{1}, \ldots, X_{r}\right)$, it follows (in characteristic 0 ) that all the $F_{i} \in I(C)$. So $I(C)$ is generated by elements which do not depend on $X_{0}$. Hence $C$ is a cone. Since $C$ is also a local complete intersection, it follows that $C$ is a complete intersection. If $C$ is a complete intersection of hypersurfaces of degrees $a_{1}, \ldots, a_{r-1}$, then $\mathscr{N}(-1) \cong \oplus_{i} \mathscr{O}_{C}\left(a_{i}-1\right)$, and $\omega_{C} \cong \mathscr{O}_{C}\left(\left(\sum_{i} a_{i}\right)-r-1\right)$. Since $H^{1}(\mathscr{N}(-1))=0$, we must have $a_{i}-1>\left(\sum_{i} a_{i}\right)-r-1$ for all $i$. It now follows that all $a_{i}$ must be 1 except possibly one which could be 2 . So $C$ is either a line or a degenerate conic as claimed. This completes the proof of the lemma.

The next result requires some background before it can be stated. Let $X \rightarrow S$ be a flat family of 1-dimensional closed subschemes of $\mathbf{P}^{r}$ of degree $d$. Let $H$ 
be a hyperplane in $\mathbf{P}^{r}$. Assume that no irreducible or embedded component of any fiber $X_{s}$ of $X \rightarrow S$ lies in $H$. Then $X \times_{\mathbf{P}^{r}} H \rightarrow S$ is a flat family of 0-dimensional subschemes $X_{s} \cap H$ of $H$ of length $d$. The universal property of the Hilbert scheme then asserts that there is a unique morphism $f: S \rightarrow$ Hilb $^{d} H$ such that $X \times_{\mathbf{p}^{r}} H \rightarrow S$ is the pullback with respect to $f$ of the universal family over $\mathrm{Hilb}^{d} H$.

We now consider $\mathrm{Hilb}^{d} H$. It can have several irreducible components, but only one concerns us. Specifically, let $L C I(d)$ be the open subscheme of $\mathrm{Hilb}^{d} \mathrm{H}$ of local complete intersection schemes. Any local complete intersection scheme of length $d$ can easily be deformed into the union of $d$ distinct points, so $L C I(d)$ is an open subscheme of the irreducible component of $\mathrm{Hilb}^{d} H$ containing the unions of $d$ distinct points. Furthermore, $L C I(d)$ is a nonsingular variety. This is because (i) there are no local obstructions to deforming local complete intersection subschemes of a regular scheme (cohomologically the higher cotangent sheaf $\mathscr{T}^{2}$, which contains the local obstructions, vanishes), and (ii) there are no global obstructions to deforming 0-dimensional subschemes (cohomologically $H^{1}(\mathscr{N})=0$ ). Thus all infinitesimal deformations of fibers of $L C I(d)$ can be extended without obstruction, implying that the local rings of $L C I(d)$ must be regular.

Finally, if $X \rightarrow S$ is a flat family of local complete intersection curves in $\mathbf{P}^{r}$ such that no fiber $X_{S}$ has an irreducible component lying in the hyperplane $H$ of $\mathbf{P}^{r}$, then the morphism $f: S \rightarrow$ Hilb $^{d} H$ described earlier factors through a map $f_{1}: S \rightarrow L C I(d)$.

Theorem 5. Let $k$ be a field of characteristic 0 . Let $d, g, r$ be integers such that $d \geq 2$ and $r \geq 4$. Let $X \rightarrow S$ be a flat family of local complete intersection curves in $\mathbf{P}_{k}^{r}$ of degree $d$ and arithmetic genus $g$. Let $H$ be a hyperplane in $\mathbf{P}_{k}^{r}$. Suppose that no irreducible component of any fiber $X_{s}$ lies in $H$. If the map $f_{1}: S \rightarrow L C I(d)$ defined above is dominant, then $g \leq(2 d-4) /(r-3)$.

Proof. It is enough to consider the case when $X \rightarrow S$ is the universal family over an open subscheme of an irreducible component of the Hilbert scheme of local complete intersection curves in $\mathbf{P}^{r}$ of degree $d$ and arithmetic genus $g$. Under that assumption, $T_{s} S \cong H^{0}\left(\mathscr{N}_{X_{s}}\right)$ for all closed points $s \in S$. Moreover, the map $f_{1}: S \rightarrow L C I(d)$ defined by $s=\left[X_{s}\right] \rightarrow\left[X_{s} \cap H\right]$ has differential $(d f)_{s}: T_{s} S \rightarrow T_{f(s)} L C I(d)$ which is the first arrow of the long exact sequence

$$
H^{0}\left(\mathscr{N}_{X_{s}}\right) \rightarrow H^{0}\left(\mathscr{N}_{X_{s} \cap H / H}\right) \rightarrow H^{1}\left(\mathscr{N}_{X_{s}}(-1)\right) \rightarrow H^{1}\left(\mathscr{N}_{X_{s}}\right)
$$

Now suppose that $f_{1}$ is dominant. Then the map of reduced induced subschemes $f_{1, \text { red }}: S_{\text {red }} \rightarrow L C I(d)$ is also dominant. (Note that $L C I(d)$ is its own reduced induced subscheme because it is smooth by the discussion preceding the statement of the theorem.) Since the characteristic is assumed to be 0 , the generic smoothness theorem applies. So at a general point $s \in S$, the differential $\left(d f_{1, \text { red }}\right)_{s}: T_{s} S_{\text {red }} \rightarrow T_{f(s)} L C I(d)$ is surjective. Then $\left(d f_{1}\right)_{s}$ must be surjective at a general $s \in S$ as well.

Returning to the exact sequence, we see that for a general $s$, the map $\alpha$ : $H^{1}\left(\mathscr{N}_{X_{s}}(-1)\right) \rightarrow H^{1}\left(\mathscr{N}_{X_{s}}\right)$ is injective. So its dual $\alpha^{*}$ is surjective. By the 
functoriality of Serre duality, $\alpha^{*}$ is the map

$$
H^{0}\left(\mathcal{N}_{X_{s}}^{\vee} \otimes \omega_{X_{s}}\right) \rightarrow H^{0}\left(\mathscr{N}_{X_{s}}^{\vee} \otimes \omega_{X_{s}}(1)\right)
$$

induced by multiplication by the linear form defining $H$. However, $\mathscr{N}_{X_{s}}^{\mathrm{V}} \otimes \omega_{X_{s}}$ is a locally free sheaf on a local complete intersection curve, so it has no 0 dimensional associated points. And it is easy to see that for a coherent sheaf $\mathscr{E}$ without 0 -dimensional associated points, the map $H^{0}(\mathscr{E}) \rightarrow H^{0}(\mathscr{E}(1))$ given by multiplication by a linear form can be surjective if and only if $H^{0}(\mathscr{E})=$ $H^{0}(\mathscr{E}(1))=0$. Thus $H^{0}\left(\mathscr{N}_{X_{s}}^{\vee} \otimes \omega_{X_{s}}(1)\right)=0$, and dually $H^{1}\left(\mathscr{N}_{X_{s}}(-1)\right)=0$. By Lemma 4, we now have either $d=1$ or $g \leq(2 d-4) /(r-3)$. This completes the proof of the theorem.

Lemma 6. Let $C$ be an arithmetically Cohen-Macaulay curve in $\mathbf{P}^{r}$ of degree $d$ and arithmetic genus $g$. Then $g \geq t d+1-\left(\begin{array}{c}t+r \\ r\end{array}\right)$ for all integers $t \geq-r$.

Proof. From the exact sequence $0 \rightarrow \mathscr{I}_{C} \rightarrow \mathscr{O}_{\mathbf{P}^{r}} \rightarrow \mathscr{O}_{C} \rightarrow 0$ we see that

$$
\chi\left(\mathscr{J}_{C}(t)\right)=\chi\left(\mathscr{O}_{\mathbf{P r}}(t)\right)-\chi\left(\mathscr{O}_{C}(t)\right)=\left(\begin{array}{c}
t+r \\
r
\end{array}\right)-(t d+1-g) .
$$

Hence the lemma is equivalent to the assertion that $\chi\left(\mathscr{I}_{C}(t)\right) \geq 0$ for all $t \geq-r$.

But since $C$ is of dimension 1, the same exact sequence shows $H^{i}\left(\mathscr{J}_{C}(t)\right)=0$ for all $t \geq-r$ unless $i \in\{0,1,2\}$. And since $C$ is arithmetically CohenMacaulay, we have $H^{1}\left(\mathscr{F}_{C}(t)\right)=0$ for all $t$ as well. Hence $\chi\left(\mathscr{I}_{C}(t)\right)=h^{0}+$ $h^{2} \geq 0$ for all $t \geq 0$. The lemma follows.

Proof of Theorem 1. If a general set of $d$ points in $\mathbf{P}^{r-1}$ were the hyperplane section of an arithmetically Cohen-Macaulay local complete intersection curve in $\mathbf{P}^{r}$, then there would exist a flat family $X \rightarrow S$ of arithmetically CohenMacaulay local complete intersection curves in $\mathbf{P}^{r}$ of the same degree $d$ and some constant arithmetic genus $g$ for which the map $f: S \rightarrow L C I(d)$ of Theorem 5 would be dominant. But then Theorem 5 and Lemma 6 would give inequalities

$$
\frac{2 d-4}{r-3} \geq g \geq t d+1-\left(\begin{array}{c}
t+r \\
r
\end{array}\right)
$$

for all integers $t \geq-r$. Solving for $d$ would give

$$
d \leq \frac{\left(\begin{array}{c}
t+r \\
r
\end{array}\right)-\frac{r+1}{r-3}}{t-\frac{2}{r-3}}
$$

for all $t>\frac{2}{r-3}$. If $r=4$, then setting $t=3$ makes (1) become $d \leq 30$. If $r=5$, then setting $t=2$ makes (1) become $d \leq 18$. If $r \geq 6$, then setting $t=1$ makes (1) become $d \leq r+2+\frac{6}{r-5}$. The asserted values of $d_{r}$ for $r \geq 6$ are the smallest integers strictly greater than $r+2+\frac{6}{r-5}$. The theorem now follows.

\section{REFERENCES}

[B] E. Ballico, Points not as hyperplane sections of projectively normal curves, Proc. Amer. Math. Soc. 112 (1991), 343-346.

[BM] E. Ballico and J. Migliore, Smooth curves whose hyperplane section is a given set of points, Comm. Algebra 18 (1990), 3015-3040. 
[CO] L. Chiantini and F. Orecchia, Plane sections of arithmetically normal curves in $\mathbf{P}^{3}$, Lecture Notes in Math., vol. 1389, Springer-Verlag, New York, 1989, pp. 32-42.

[EEK] L. Ein, D. Eisenbud, and S. Katz, Varieties cut out by quadrics: Scheme-theoretic versus homogeneous generation of ideals, Lecture Notes in Math., vol. 1311, Springer-Verlag, New York, 1988, pp. 51-70.

[GP] L. Gruson and C. Peskine, Genre des courbes de l'espace projectif, Lecture Notes in Math., vol. 687, Springer-Verlag, New York, 1978, pp. 31-50.

[H] R. Hartshorne, Algebraic geometry, Springer-Verlag, New York, 1977.

[RR] L. Reid and L. Roberts, Intersection points of seminormal configurations of lines, Contemp. Math., vol. 126, Amer. Math. Soc., Providence, RI, 1993, pp. 151-163.

[W] C. Walter, Hyperplane sections of curves of small genus, Comm. Algebra 22 (1994), 51675174.

Département de Mathématiques, Université de Nice, Parc Valrose, 06108 Nice Cedex 02, France

E-mail address: walter@math.unice.fr 